\title{
Autopercepção de saúde em adolescentes, adultos e idosos
}

\author{
Self-reported health status in adolescents, adults and the elderly
}

Felipe Fossati Reichert ${ }^{1}$

Mathias Roberto Loch ${ }^{2}$

Marcelo Fernandes Capilheira ${ }^{3}$

\footnotetext{
${ }^{1}$ Grupo de Estudos em Epidemiologia da Atividade Física, Escola Superior de Educação Física,

Universidade Federal de Pelotas. Rua Luis de Camões 625, Três Vendas. 96055-630 Pelotas RS. ffreichert@gmail.com ${ }^{2}$ Programa de PósGraduação em Saúde Coletiva, Departamento de Educação Física,

Universidade Estadual de Londrina.

${ }^{3}$ Faculdade de Medicina, Universidade Federal de Pelotas.
}

\begin{abstract}
The scope of this study was to verify the prevalence and associated factors of self-reported health status as regular/bad. A cross-sectional, population-based study was conducted in Pelotas. The sample size comprised 820 adolescents, 2715 adults and 385 elderly. Self-reported health status was investigated via the question: "How do you rate your health?" Data on demographics, socioeconomic, behavioral, and health-related characteristics of individuals were gathered. Adjusted prevalence ratios were estimated through the Poisson regression. Prevalence of reporting health status as regular or bad was $12.1 \%, 22.3 \%$ and $49.4 \%$ in adolescents, adults and the elderly, respectively. Adolescents with lower economic status and schooling had higher prevalence of regular/bad selfreported health. Among adults and the elderly, women and older men with lower economic status and some morbidity presented a higher proportion of regular/bad self-reported health. In conclusion, individuals perceive health not only as the absence of a disease, but also as a construct related to social, demographic and, to a lesser extent, behavioral aspects. Health approaches must recognize this fact and transcend the simplistic model where health is dichotomized into the sick and the non-sick.
\end{abstract}

Key words Self-reported health status, Prevalence, Associated factors, Risk factors, Epidemiology
Resumo O objetivo deste estudo foi verificar a prevalência e os fatores associados a autopercepção de saúde regular/ruim em adolescentes, adultos e idosos. O estudo foi transversal, de base populacional, realizado em Pelotas. A amostra compreendeu 820 adolescentes, 2715 adultos e 385 idosos. Autopercepção de saúde foi investigada pela pergunta: "Como o Sr(a) considera sua saúde?" Características demográficas, socioeconômicas, comportamentais e de saúde foram coletadas. Razões de prevalência ajustada foram estimadas pela Regressão de Poisson. A prevalência de autopercepção regular ou ruim de saúde foi de 12,1\%, 22,3\% e 49,4\% entre adolescentes, adultos e idosos, respectivamente. Adolescentes com menor nível econômico e com escolaridade não adequada relataram pior autopercepção de saúde. Entre os adultos $e$ idosos, relataram pior autopercepção de saúde: as mulheres, aqueles com maior faixa etária, menor nivel econômico e os que possuíam alguma morbidade. Conclui-se que a população percebe saúde não apenas como ausência de doença, mas também como um constructo relacionado com aspectos sociais e demográficos, e em menor magnitude, com aspectos comportamentais. Abordagens em saúde devem superar o modelo simplista onde saúde é dicotomizada em doente e não-doente.

Palavras-chaves Autopercepção de saúde, Prevalência, Fatores associados, Fatores de risco, Epidemiologia 


\section{Introdução}

A percepção subjetiva da saúde tem sido explorada em estudos epidemiológicos. Tal variável é simples de ser obtida e fornece informações importantes acerca da população estudada. Pesquisas demonstram associação entre esta medida subjetiva e indicadores objetivos de saúde, tanto em delineamentos transversais, quanto longitudinais $^{1-4}$.

Considerando que aspectos culturais, psicológicos, sociais, entre outros, podem influenciar a percepção de saúde dos indivíduos, é razoável ter como hipótese que, especialmente em países em desenvolvimento, onde há forte desigualdade social, as diferenças econômicas e sociais, e até mesmo comportamentais, podem produzir padrões de autopercepção de saúde diferenciados entre sujeitos com diferentes condições e características. Estudo realizado na Suécia ${ }^{5}$ demonstrou que a autopercepção de saúde variou de acordo com a etnia, e que essas diferenças foram consideravelmente reduzidas após controle por fatores econômicos e psicossociais, sugerindo que estes sejam importantes determinantes da autopercepção de saúde.

No Brasil, estudo por inquérito telefônico, realizado em todas as capitais brasileiras, observou que a prevalência de sujeitos que referiram sua condição de saúde como ruim (em uma escala que incluía as opções: excelente, boa, regular e ruim) variou de 3,0\% em Belo Horizonte (Minas Gerais) a 7,1\% em Salvador (Bahia) ${ }^{6}$. Ainda no contexto brasileiro, investigação com trabalhadores da indústria de Santa Catarina observou que mulheres, pessoas com maior faixa etária, menor renda familiar e escolaridade, que não praticavam atividade física no lazer e que referiram uma pior percepção da qualidade do sono e de estresse, apresentavam maior prevalência de percepção negativa de saúde ${ }^{2}$. Ainda no Brasil, outros trabalhos investigaram especificamente a percepção em adolescentes ${ }^{7-9}$ e idosos ${ }^{10,11}$.

Considerando que os fatores associados à autopercepção de saúde podem diferir segundo faixa etária, os objetivos deste estudo foram: a) explorar a autopercepção de saúde em adolescentes, adultos e idosos e b) estudar a associação entre variáveis demográficas, socioeconômicas, comportamentais e indicadoras de morbidade com autopercepção de saúde regular/ruim.

\section{Metodologia}

Foi realizado um estudo transversal na cidade de Pelotas, localizada no extremo sul do Brasil. A amostra, representativa da área urbana, foi selecionada em dois estágios. Primeiramente, todos os setores censitários da área urbana foram listados $(\mathrm{n}=404)$ de acordo com a renda mensal do chefe de família. Destes, 144 foram selecionados com probabilidade proporcional ao tamanho. Dentro de cada setor selecionado, adotouse uma estratégia sistemática para seleção de 10 domicílios. Todos os moradores de 10 anos ou mais residentes nos domicílios amostrados eram elegíveis para o estudo.

Cálculos de tamanho de amostra foram realizados tendo como base o menor grupo etário estudado (idosos). As análises realizadas têm poder de pelo menos $80 \%$ para detectar razões de prevalência de 1,50 , estimando-se uma prevalência do desfecho no grupo exposto de $45 \%$, frequência de exposição de 50\%.

A variável dependente (autopercepção de saúde) foi avaliada pela questão: Como você considera a sua saúde? As seguintes opções de resposta foram lidas para o entrevistado: excelente, muito boa, boa, regular e ruim. Para fins de análise, as categorias foram reagrupadas, dicotomicamente, em regular/ruim e excelente/muito boa/boa.

As variáveis exploratórias investigadas dentro de cada faixa etária (adolescentes - 10 a 19 anos, adultos -20 a 64 anos e idosos $-\geq 65$ anos) foram agrupadas em demográficas, socioeconômicas, comportamentais e de morbidade. Dentre as demográficas, investigou-se sexo, idade, cor da pele e religião nos três grupos etários e situação conjugal em adultos e idosos. O nível econômico e a escolaridade foram investigados em toda a amostra. Tabagismo (investigado nos três grupos etários) e prática de atividade física no lazer (investigada apenas entre adultos e idosos) foram as variáveis comportamentais exploradas. Em relação às morbidades, o estado nutricional foi investigado nos três grupos etários, enquanto que osteoporose, hipertensão, diabetes, doença cardíaca, doença crônica do pulmão, e câncer foram investigadas apenas em adultos e idosos. As variáveis de morbidade (exceto obesidade) foram investigadas através do autorrelato de diagnóstico médico.

A cor da pele foi definida de acordo com a observação do entrevistador (e posteriormente dicotomizada em branca ou não branca). O nível econômico foi estimado segundo critério de classificação Econômica Brasil ${ }^{12}$, a qual conside- 
ra a escolaridade do chefe da família, número de determinados eletrodomésticos no domicílio e presença de empregado mensalista na residência para classificar as famílias em níveis A (mais alto), B, C, D ou E (mais baixo). Entre os adolescentes, esta variável foi recategorizada em grupos A/B, C, D/E em virtude do baixo número de famílias nos extremos. A escolaridade foi avaliada a partir dos anos completos de estudo em adultos e idosos e entre adolescentes foi construída uma variável indicativa de escolaridade adequada ou inadequada para a idade. Escolaridade inadequada foi considerada quando com dez anos de idade o adolescente ainda não houvesse concluído a segunda série do ensino fundamental, com 11 anos a terceira série e assim sucessivamente. Aqueles que fumaram pelo menos um cigarro ao dia por pelo menos um mês foram considerados fumantes. A prática de atividades físicas no lazer foi investigada em adultos e idosos pela seção de lazer da versão longa do Questionário Internacional de Atividade Física (IPAQ) ${ }^{13}$. Foram considerados praticantes de atividade física aqueles que atingiram $150 \mathrm{~min} / \mathrm{sem}$ de atividades físicas moderadas ou vigorosas, sendo que o tempo em atividades vigorosas foi multiplicado por dois. $\mathrm{O}$ estado nutricional foi avaliado pelo índice de massa corporal (IMC), o qual é calculado pelo quociente do peso $(\mathrm{kg})$ pela estatura $(\mathrm{m})$ ao quadrado. Adolescentes tiveram o peso e estatura mensurados, enquanto que em adultos e idosos estas variáveis foram autorrelatadas. A classificação do estado nutricional seguiu as recomendações da Organização Mundial da Saúde ${ }^{14}$. Adolescentes acima do percentil 85 para idade e sexo foram classificados como tendo excesso de peso e adultos e idosos acima de $24,9 \mathrm{~kg} / \mathrm{m}^{2}$ e $29,9 \mathrm{~kg} /$ $\mathrm{m}^{2}$ foram classificados como tendo sobrepeso e obesidade, respectivamente.

Os dados foram coletados por entrevistadoras selecionadas após treinamento. Indivíduos que não foram entrevistados após pelo menos três visitas da entrevistadora e uma de um supervisor de campo, foram considerados como perdas ou recusas. Os questionários foram codificados, revisados e duplamente digitados no programa Epi-Info 6.04d. A análise foi realizada através do programa Stata 9.2.

Para a estimativa de razões de prevalência ajustada, foi utilizada a regressão de Poisson. Esta análise multivariável seguiu um modelo hierárquico com as variáveis demográficas e socioeconômicas em um nível distal, as comportamentais no nível intermediário e as variáveis de morbidade no nível proximal. Ajustes foram realiza- dos para variáveis do mesmo nível ou nível mais distal que apresentaram um valor $\mathrm{p}<0,2$. Entre os adultos foi explorada a associação entre a idade e o desfecho. Análise equivalente para adolescentes e idosos não foi conduzida devido à baixa heterogeneidade da idade. Todas as análises levaram em consideração o efeito de delineamento.

O comitê de Ética da Faculdade de Medicina da Universidade Federal de Pelotas aprovou o projeto deste estudo e consentimentos informados foram obtidos de cada participante.

\section{Resultados}

O número de adolescentes, adultos e idosos avaliados foi, respectivamente, de 820, 2715 e 385 . Considerando toda a amostra, a maioria foi composta por mulheres $(55,3 \%)$, brancos $(79,4 \%)$ e foi classificada nos grupos econômicos C, D e E $(74,8 \%)$. O percentual de perdas e recusas totalizou 3,5\%. Outras características da amostra são apresentadas nas Tabelas 1 (adolescentes), 2 (adultos) e 3 (idosos).

A Figura 1 apresenta a distribuição das respostas para a pergunta referente à autopercepção de saúde para os três grupos etários estudados. Observa-se uma associação positiva da autopercepção de saúde regular/ruim de acordo com o avançar da idade. Nos adolescentes, 12,1\% $\left(\mathrm{IC}_{95 \%}: 9,9 \%-14,4 \%\right)$ apresentaram autopercepção de saúde regular/ruim, enquanto que entre adultos esta proporção foi de $22,3 \%$ ( IC $_{95 \%}$ : $20,8 \%-23,9 \%)$ e entre idosos de $49,4 \%\left(\right.$ IC $_{95 \%}$ : $44,3 \%-54,4 \%$ ) (Figura 1).

A Tabela 1 também apresenta a associação bruta e a ajustada entre autopercepção de saúde e variáveis independentes nos adolescentes. $\mathrm{Na}$ análise ajustada, o nível econômico e a escolaridade estiveram inversamente associados com o desfecho. Sexo, cor da pele, religião e IMC não estiveram associadas com autopercepção de saúde. Na análise bruta, a prevalência de autopercepção de saúde regular/ruim foi superior entre fumantes comparado aos não fumantes, mas essa associação perdeu significância na análise ajustada.

A Tabela 2 apresenta a associação entre autopercepção de saúde e exposições nos adultos. Variáveis demográficas (sexo e idade), socioeconômicas (nível econômico e escolaridade), comportamentais (tabagismo) e de morbidade (hipertensão, diabetes, doença cardíaca, doença crônica do pulmão e câncer) estiveram associadas com o desfecho nas análises bruta e ajustada. 


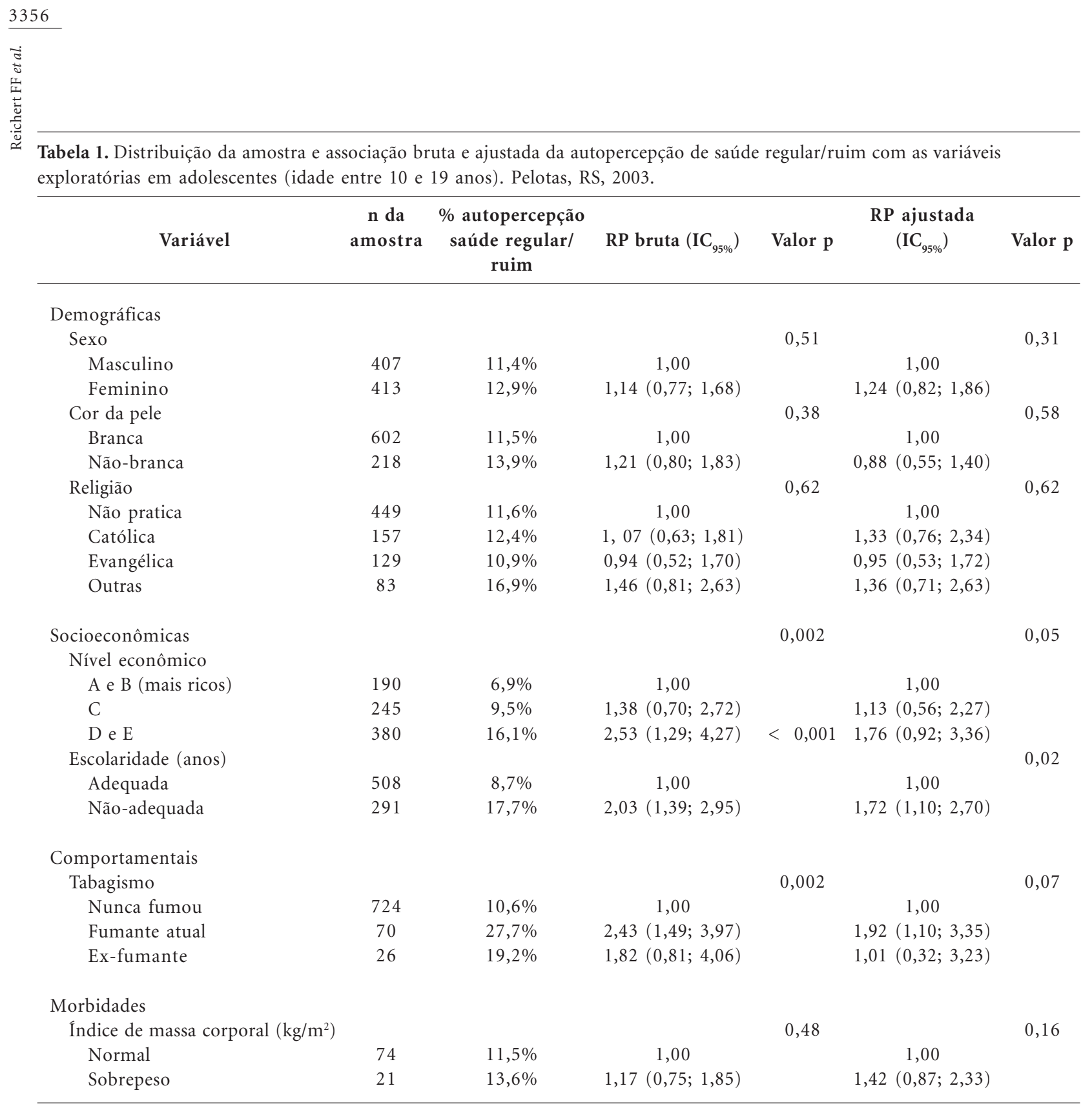

A Tabela 3 apresenta a associação entre autopercepção de saúde e exposições nos idosos. De maneira geral, as associações foram similares às observadas com adultos. Prevalência superior de autopercepção de saúde regular/ruim foi observada em mulheres e aqueles com presença de determinadas morbidades. Além disso, quanto maior a escolaridade, menor a prevalência de autopercepção de saúde regular/ruim.

A Figura 2 demonstra uma forte associação linear entre número de morbidades relatadas e autopercepção de saúde em adultos e idosos: quanto maior o número de morbidades relatadas, maior a prevalência de percepção de saúde regular/ruim.

\section{Discussão}

Este estudo investigou a autopercepção de saúde em adolescentes, adultos e idosos, de Pelotas, cidade do sul do Brasil. A inclusão de variáveis desta natureza, em estudos epidemiológicos, é importante, haja vista a necessidade de se entender a saúde como uma condição complexa e multifatorial e, mais do que isso, compreender a dimensão subjetiva da saúde humana. A similaridade da distribuição de variáveis demográficas e econômicas entre amostra e os dados do censo do IBGE para a cidade ${ }^{15}$, bem como os procedimentos metodológicos adotados evidenciam a representatividade do estudo. 
Tabela 2. Distribuição da amostra e associação bruta e ajustada da autopercepção de saúde regular/ruim com as variáveis exploratórias em adultos (idades entre 20 e 64 anos). Pelotas, RS, 2003.

\begin{tabular}{|c|c|c|c|c|c|c|}
\hline Variável & $\begin{array}{c}\mathrm{n} \mathrm{da} \\
\text { amostra }\end{array}$ & $\begin{array}{c}\text { \% autopercepção } \\
\text { saúde regular/ } \\
\text { ruim }\end{array}$ & RP bruta $\left(\mathrm{IC}_{95 \%}\right)$ & Valor $\mathrm{p}$ & $\begin{array}{l}\text { RP ajustada } \\
\quad\left(\mathrm{IC}_{95 \%}\right)\end{array}$ & Valor $\mathbf{p}$ \\
\hline \multicolumn{7}{|l|}{ Demográficas } \\
\hline Sexo & & & & $<0,001$ & & $<0,001$ \\
\hline Masculino & 1202 & $17,3 \%$ & 1,00 & & 1,00 & \\
\hline Feminino & 1513 & $26,3 \%$ & $1,52(1,32 ; 1,76)$ & & $1,58(1,34 ; 1,87)$ & \\
\hline Idade (anos) & & & & $<0,001$ & & $<0,001$ \\
\hline $20-29$ & 759 & $10,6 \%$ & 1,00 & & 1,00 & \\
\hline $30-39$ & 645 & $18,9 \%$ & $1,79(1,37 ; 2,33)$ & & $1,59(1,20 ; 2,12)$ & \\
\hline $40-49$ & 680 & $25,8 \%$ & $2,44(1,94 ; 3,07)$ & & $2,18(1,66 ; 2,85)$ & \\
\hline $50-64$ & 631 & $36,2 \%$ & $3,42(2,77 ; 4,22)$ & & $2,74(2,10 ; 3,59)$ & \\
\hline Cor da pele & & & & 0,01 & & 0,88 \\
\hline Branca & 2191 & $21,3 \%$ & 1,00 & & 1,00 & \\
\hline Não-branca & 524 & $26,6 \%$ & $1,25(1,05 ; 1,49)$ & & $1,01(0,83 ; 1,23)$ & \\
\hline Religião & & & & $<0,001$ & & 0,88 \\
\hline Não pratica & 1245 & $18,7 \%$ & 1,00 & & 1,00 & \\
\hline Católica & 699 & $24,4 \%$ & $1,30(1,07 ; 1,58)$ & & $1,04(0,84 ; 1,28)$ & \\
\hline Evangélica & 399 & $31,1 \%$ & $1,66(1,33 ; 2,06)$ & & $1,10(0,87 ; 1,37)$ & \\
\hline Outras & 368 & $21,5 \%$ & $1,15(0,89 ; 1,48)$ & & $1,00(0,77 ; 1,30)$ & \\
\hline Situação conjugal & & & & 0,09 & & 0,25 \\
\hline Casado ou com companheiro & 1777 & $23,2 \%$ & 1,00 & & 1,00 & \\
\hline Outro & 938 & $20,6 \%$ & $0,89(0,77 ; 1,02)$ & & $0,90(0,76 ; 1,08)$ & \\
\hline \multicolumn{7}{|l|}{ Socioeconômicas } \\
\hline Nível econômico & & & & $<0,001$ & & $<0,001$ \\
\hline A (mais ricos) & 138 & $7,3 \%$ & 1,00 & & 1,00 & \\
\hline $\mathrm{B}$ & 566 & $12,4 \%$ & $1,71(0,96 ; 3,04)$ & & $1,26(0,65 ; 2,46)$ & \\
\hline $\mathrm{C}$ & 899 & $19,7 \%$ & $2,72(1,60 ; 4,61)$ & & $1,66(0,86 ; 3,20)$ & \\
\hline $\mathrm{D}$ & 935 & $30,9 \%$ & $4,27(2,56 ; 7,13)$ & & $2,05(1,05 ; 4,01)$ & \\
\hline $\mathrm{E}$ & 165 & $33,9 \%$ & $4,68(2,70 ; 8,14)$ & & $2,11(1,03 ; 4,36)$ & \\
\hline Escolaridade (anos) & & & & $<0,001$ & & $<0,001$ \\
\hline$\geq 12$ & 443 & $9,5 \%$ & 1,00 & & 1,00 & \\
\hline$\overline{9}-11$ & 776 & $10,6 \%$ & $1,12(0,73 ; 1,71)$ & & $1,09(0,74 ; 1,60)$ & \\
\hline $5-8$ & 913 & $26,3 \%$ & $2,77(1,98 ; 3,88)$ & & $2,16(1,51 ; 3,10)$ & \\
\hline $0-4$ & 583 & $41,6 \%$ & $4,39(3,16 ; 6,08)$ & & $2,76(1,88 ; 4,06)$ & \\
\hline \multicolumn{7}{|l|}{ Comportamentais } \\
\hline Tabagismo & & & & $<0,001$ & & 0,004 \\
\hline Nunca fumou & 1363 & $17,5 \%$ & 1,00 & & 1,00 & \\
\hline Fumante atual & 782 & $29,3 \%$ & $1,66(1,39 ; 1,97)$ & & $1,37(1,14 ; 1,66)$ & \\
\hline Ex-fumante & 570 & $24,6 \%$ & $1,40(1,15 ; 1,71)$ & & $1,20(0,97 ; 1,48)$ & \\
\hline Prática de atividade física & & & & $<0,001$ & & 0,51 \\
\hline Sim & 743 & $16,6 \%$ & 1,00 & & 1,00 & \\
\hline Não & 1963 & $24,5 \%$ & $1,48(1,23 ; 1,77)$ & & $1,07(0,87 ; 1,31)$ & \\
\hline
\end{tabular}

Este estudo demonstrou que as mulheres adultas e idosas percebem de maneira mais negativa a sua saúde do que os homens. Estes resultados são similares aos encontrados com trabalhadores da indústria do estado de Santa Catarina ${ }^{2}$ e também com o relatório Vigitel $^{6}$, que mostrou que, nas capitais brasileiras, $6,7 \%$ das mulheres referiram como ruim o seu estado de saúde, contra 2,7\% dos homens. Alves e Rodrigues $^{10}$ verificaram, na análise bruta, que as idosas apresentaram maior frequência de autopercepção de saúde ruim em comparação aos ido- 


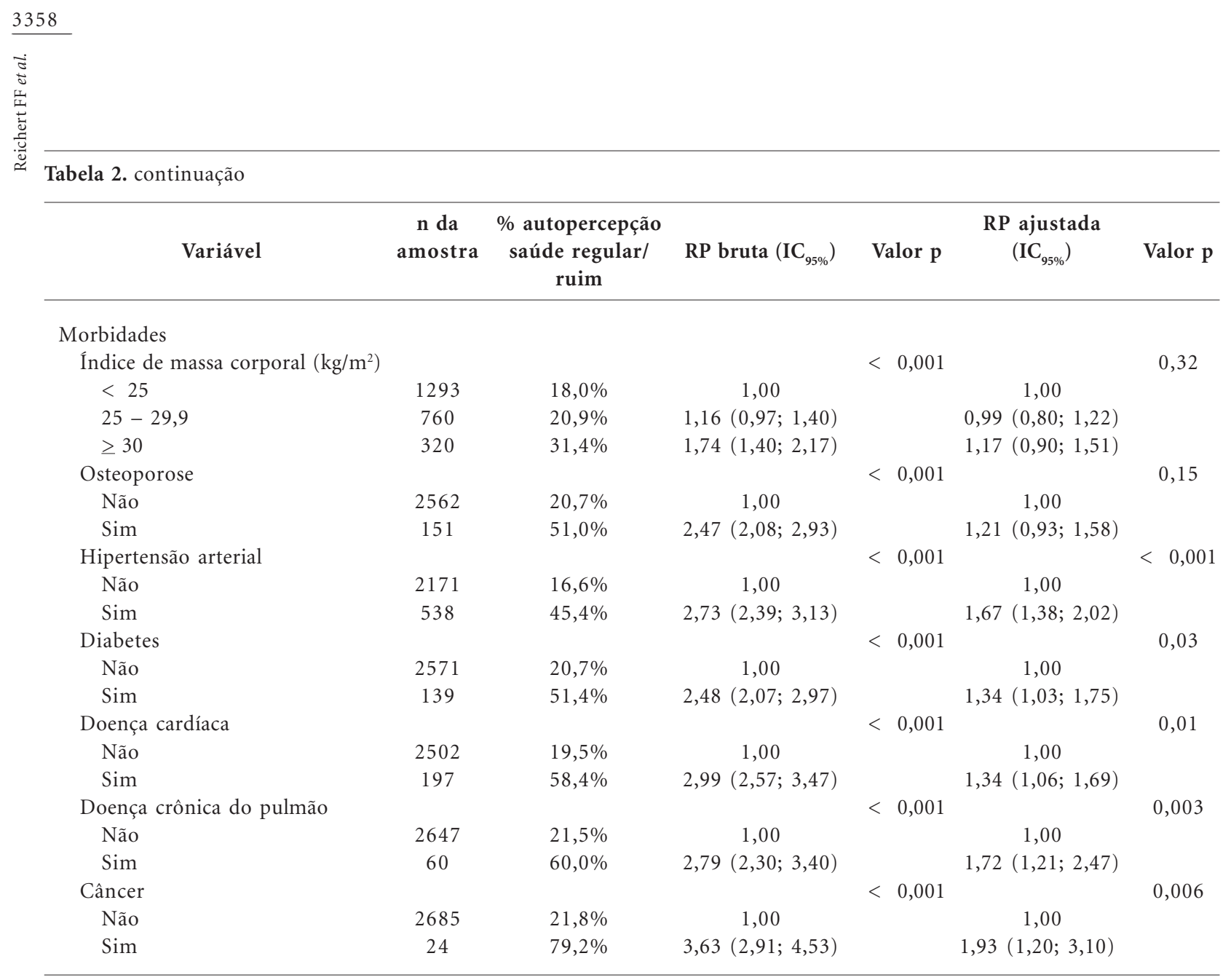

sos. Porém, destaca-se que a construção dos modelos estatísticos no estudo de Alves e Rodrigues ${ }^{10}$ e no presente estudo foi diferente. Enquanto no referido trabalho ${ }^{10} \mathrm{o}$ efeito de sexo sobre o desfecho foi verificado com a presença de mediadores na análise multivariável (presença de morbidades), no presente estudo apenas fatores de confusão foram considerados. Todavia, para fins de comparação, analisamos o efeito de sexo incluindo as variáveis de morbidade no modelo estatístico e os resultados continuaram indicando maior chance de idosas perceberem a saúde como regular/ ruim comparado aos idosos. Outros estudos são necessários para compreender melhor a relação entre sexo, presença de morbidades e autopercepção de saúde. Entre os adolescentes, este estudo não constatou diferença na autopercepção de saúde conforme sexo, diferentemente de outros estudos envolvendo adolescentes, onde as moças apresentaram uma pior percepção de saúde do que os rapazes ${ }^{7,8}$.

A cor da pele esteve associada com a percepção de saúde somente entre os adultos, na análi- se bruta. $\mathrm{Na}$ análise ajustada, nenhuma associação foi observada, reforçando a hipótese que os melhores indicadores de saúde apresentados por pessoas de pele branca são mediados por questões culturais e sociais, que quando controladas, fazem diminuir as diferenças. Estudos demonstraram que indivíduos de cor da pele branca em geral apresentam melhor condição social e econômica, têm maior acesso a serviços de saúde e apresentam menor prevalência de determinados comportamentos negativos relacionados à saú$\mathrm{de}^{16,17}$. De qualquer modo, este tema precisa ser aprofundado, inclusive para contribuir com a redução destas iniquidades.

Os dados obtidos neste estudo também possibilitaram explorar a relação entre idade e autopercepção de saúde. Quanto mais velho o grupo etário, maior foi a prevalência de autopercepção regular/ruim de saúde. Considerando apenas os adultos (idade entre 20 e 64 anos), entre os quais igualmente foi feita a divisão por idade, esta relação também ficou evidenciada, sendo que melhor percepção foi observada entre os adultos de 
Tabela 3. Distribuição da amostra e associação bruta e ajustada da autopercepção de saúde regular/ruim com as variáveis exploratórias em idosos (idade $\geq 65$ anos). Pelotas, RS, 2003.

\begin{tabular}{|c|c|c|c|c|c|c|}
\hline Variável & $\begin{array}{c}\mathrm{N} \text { da } \\
\text { amostra }\end{array}$ & $\begin{array}{c}\text { \% autopercepção } \\
\text { saúde regular/ } \\
\text { ruim }\end{array}$ & RP bruta $\left(\mathrm{IC}_{95 \%}\right)$ & Valor $\mathrm{p}$ & $\begin{array}{l}\text { RP ajustada } \\
\quad\left(\mathrm{IC}_{95 \%}\right)\end{array}$ & Valor $p$ \\
\hline \multicolumn{7}{|l|}{ Demográficas } \\
\hline Sexo & & & & 0,006 & & 0,07 \\
\hline Masculino & 142 & $39,4 \%$ & 1,00 & & 1,00 & \\
\hline Feminino & 243 & $55,2 \%$ & $1,40(1,11 ; 1,77)$ & & $1,34(0,98 ; 1,83)$ & \\
\hline Cor da pele & & & & 0,79 & & 0,97 \\
\hline Branca & 321 & $49,1 \%$ & 1,00 & & 1,00 & \\
\hline Não-branca & 64 & $50,8 \%$ & $1,04(0,80 ; 1,34)$ & & $1,01(0,68 ; 1,49)$ & \\
\hline Religião & & & & 0,63 & & 0,72 \\
\hline Não pratica & 110 & $52,3 \%$ & 1,00 & & 1,00 & \\
\hline Católica & 162 & $48,5 \%$ & $0,93(0,66 ; 1,30)$ & & $0,89(0,63 ; 1,26)$ & \\
\hline Evangélica & 65 & $51,6 \%$ & $0,99(0,64 ; 1,51)$ & & $0,88(0,57 ; 1,36)$ & \\
\hline Outras & 47 & $41,3 \%$ & $0,79(0,47 ; 1,33)$ & & $0,74(0,44 ; 1,25)$ & \\
\hline Situação conjugal & & & & 0,66 & & 0,49 \\
\hline Casado ou com companheiro & 185 & $48,1 \%$ & 1,00 & & 1,00 & \\
\hline Outro & 200 & $50,5 \%$ & $1,05(0,84 ; 1,31)$ & & $0,89(0,65 ; 1,23)$ & \\
\hline \multicolumn{7}{|l|}{ Socioeconômicas } \\
\hline Nível econômico & & & & 0,002 & & 0,47 \\
\hline A (mais ricos) & 11 & $18,2 \%$ & 1,00 & & 1,00 & \\
\hline $\mathrm{B}$ & 60 & $43,3 \%$ & $2,38(1,03 ; 5,52)$ & & $2,13(0,50 ; 9,12)$ & \\
\hline $\mathrm{C}$ & 118 & $45,8 \%$ & $2,52(1,10 ; 5,75)$ & & $2,01(0,47 ; 8,57)$ & \\
\hline $\mathrm{D}$ & 158 & $54,8 \%$ & $3,02(1,33 ; 6,84)$ & & $2,27(0,53 ; 9,72)$ & \\
\hline E & 34 & $57,6 \%$ & $3,17(1,36 ; 7,36)$ & & $2,23(0,49 ; 10,21)$ & \\
\hline Escolaridade (anos) & & & & 0,002 & & 0,03 \\
\hline$\geq 12$ & 15 & $33,3 \%$ & & & & \\
\hline $9-11$ & 40 & $27,5 \%$ & 1,00 & & 1,00 & \\
\hline $5-8$ & 100 & $48,5 \%$ & $0,83(0,29 ; 2,33)$ & & $0,78(0,27 ; 2,26)$ & \\
\hline \multirow[t]{2}{*}{$0-4$} & 228 & $55,1 \%$ & $1,45(0,66 ; 3,21)$ & & $1,36(0,54 ; 3,43)$ & \\
\hline & & & $1,65(0,76 ; 3,61)$ & & $1,53(0,63 ; 3,77)$ & \\
\hline \multicolumn{7}{|l|}{ Comportamentais } \\
\hline Tabagismo & & & & 0,48 & & 0,44 \\
\hline Nunca fumou & 224 & $49,6 \%$ & 1,00 & & 1,00 & \\
\hline Fumante atual & 44 & $56,8 \%$ & $1,15(0,86 ; 1,54)$ & & $1,32(0,84 ; 2,09)$ & \\
\hline Ex-fumante & 117 & $46,2 \%$ & $0,93(0,74 ; 1,17)$ & & $1,17(0,81 ; 1,69)$ & \\
\hline Prática de atividade física & & & & 0,05 & & 0,36 \\
\hline Sim & 84 & $39,3 \%$ & 1,00 & & 1,00 & \\
\hline Não & 300 & $52,0 \%$ & $1,32(1,00 ; 1,75)$ & & $1,19(0,82 ; 1,74)$ & \\
\hline
\end{tabular}

menor faixa etária (20 a 29 anos). Estes achados são similares aos de Fonseca et al. ${ }^{2}$, em cujo estudo a prevalência de percepção negativa de saúde foi menor entre os sujeitos com até 29 anos de idade, e maior entre os que tinham 40 ou mais anos e com os dados do Vigitel ${ }^{6}$, que constatou aumento na proporção de indivíduos que referiram percepção ruim de saúde com o aumento da idade. Esta associação forte e positiva entre idade e autopercepção de saúde regular/ruim é coerente com a noção que a incidência de doenças aumenta com o avançar da idade, e os indivíduos associam, pelo menos parcialmente, saúde com presença ou ausência de doenças.

Com relação ao nível econômico, constatouse que aqueles com o menor foram os que perceberam de maneira mais negativa a sua saúde. Do mesmo modo, aqueles com menor escolaridade 


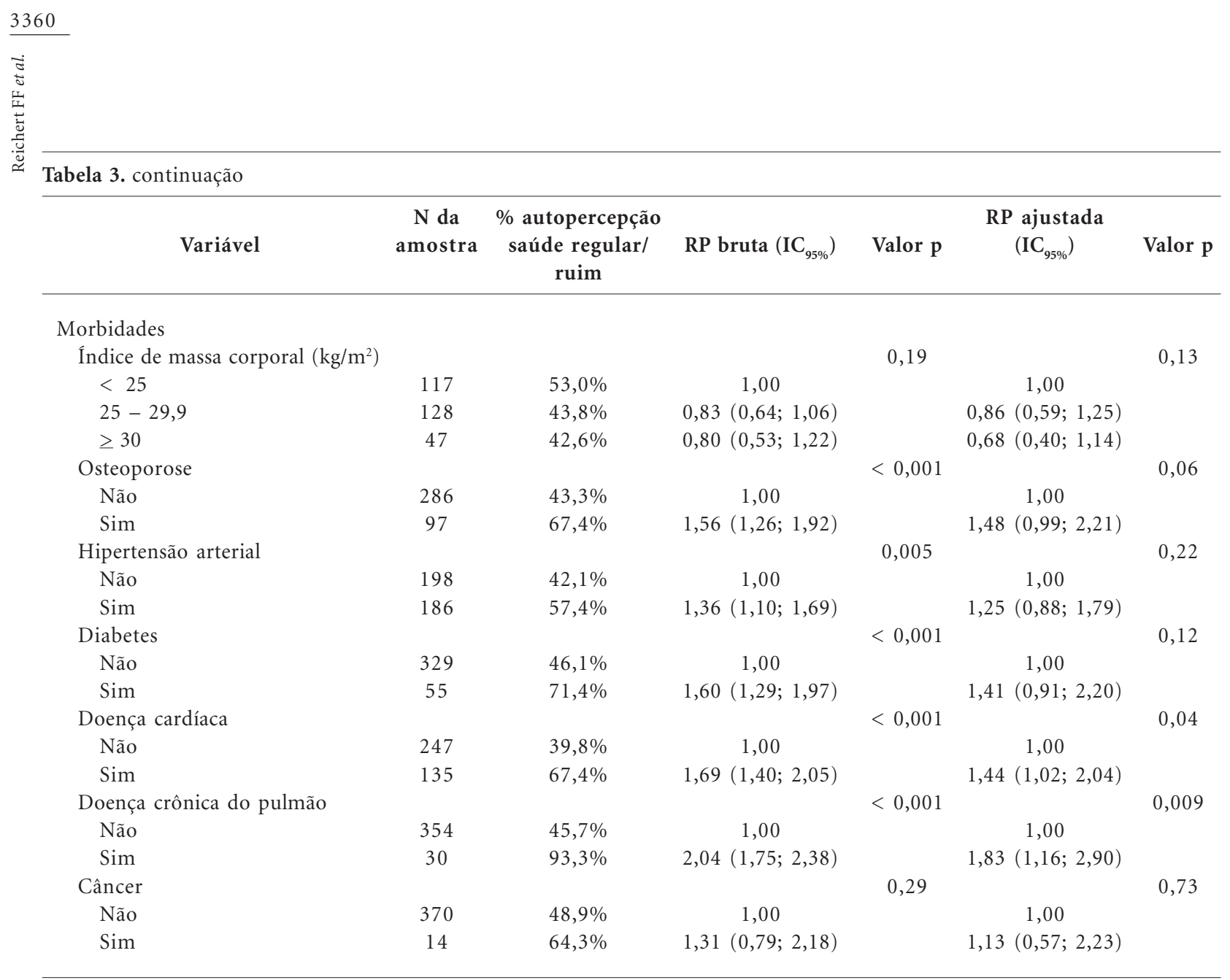

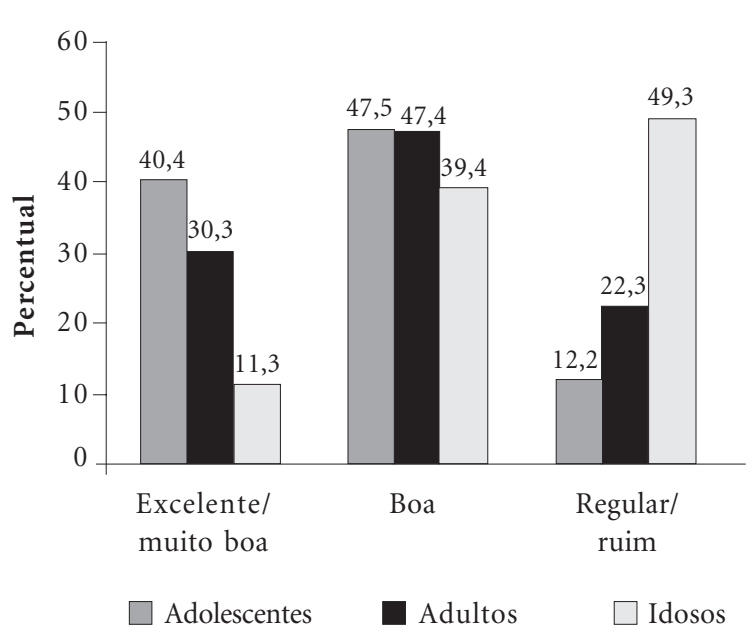

Figura 1. Distribuição da autopercepção de saúde em adolescentes, adultos e idosos. Pelotas, RS, 2003.

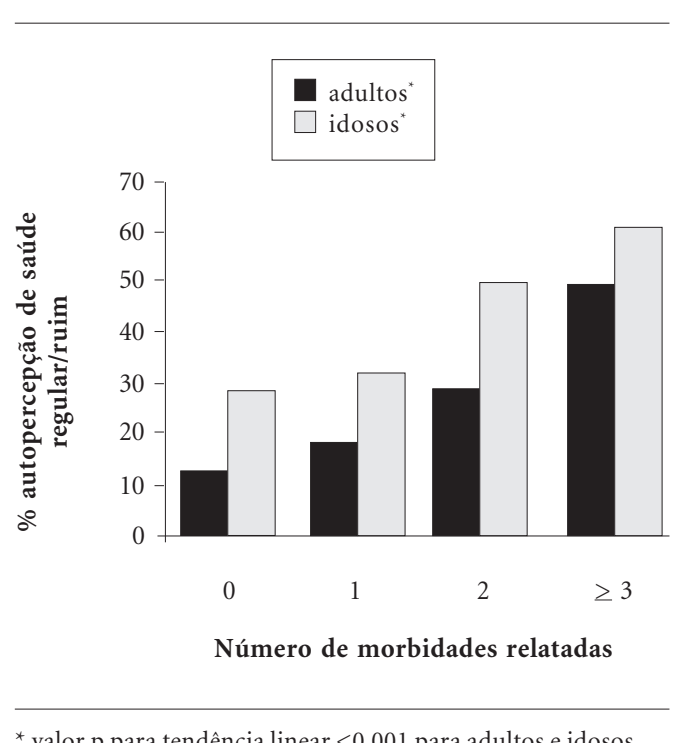

Figura 2. Prevalência da autopercepção de saúde regular/ruim em adultos e idosos de acordo com o número de morbidades relatadas. Pelotas, RS, 2003. 
apresentaram este mesmo padrão com o desfecho investigado. Resultados similares foram encontrados por Hernàndez et al. ${ }^{18}$, Vigitel ${ }^{6}$ e Alves e Rodrigues ${ }^{10}$ e reforçam a importância dos determinantes sociais e econômicos como aspectos de grande influência na determinação da saúde de pessoas e populações, não apenas nos indicadores objetivos, mas também envolvendo a percepção dos indivíduos.

A prevalência de autopercepção regular/ruim de saúde foi maior entre hipertensos e cardiopatas. Após controle para fatores de confusão, a variável diabetes perdeu significância estatística. Pode-se observar que as razões de prevalências das três morbidades na análise ajustada foram similares. Além disso, cerca de dois terços dos indivíduos diabéticos também apresentaram hipertensão. Ao se repetir a análise sem controlar para a variável hipertensão, a diabetes esteve significativamente associada à autopercepção de saúde (dados não apresentados).

Com relação ao IMC, nenhuma associação na análise ajustada foi observada com o desfecho. Este resultado é diferente do encontrado entre industriários do estado de $\mathrm{SC}^{2}$, onde foi constatado que os sujeitos nos extremos da distribuição do IMC (ou seja, que apresentavam baixo peso ou obesidade) apresentavam pior autopercepção de saúde do que os indivíduos nas categorias intermediárias de IMC (sujeitos com peso normal, ou mesmo com sobrepeso). Deve-se observar que indivíduos com IMC acima de 30kg/ $\mathrm{m}^{2}$ no presente estudo também apresentaram maior prevalência de autopercepção de saúde regular/ruim, perdendo significância apenas na análise multivariável, devido principalmente ao ajuste para presença de morbidades (fator não investigado no estudo com industriários de SC).

Nas variáveis comportamentais, vale destacar que o tabagismo se mostrou associado à autopercepção de saúde (exceção feita aos idosos). Entre adolescentes e entre adultos foi constatada pior autopercepção de saúde entre os fumantes, o que reforça a idéia que atualmente a maior parte da população interpreta o hábito de fumar como algo negativo em relação à saúde, mesmo que muitos ainda apresentem dificuldades para abandonar este hábito.

A questão utilizada para determinação de autopercepção de saúde no presente estudo utili- zou como opções de resposta uma escala com cinco categorias: excelente, muito boa, boa, regular ou ruim. A opção por uma das categorias é subjetiva e pode estar fortemente associada ao entendimento que o indivíduo tem de saúde. Estudos recentes avançam neste aspecto, e buscam compreender o que indivíduos com diferentes idades e condições entendem por saúde ${ }^{19,20}$. Além do mais, vale mencionar que a categoria "percepção" é utilizada - e pode assumir diferentes significados - em áreas do conhecimento distintas. No presente estudo assumiu-se que é através da percepção do indivíduo que este organiza e interpreta suas impressões sensoriais. Assim, acredita-se que a autoavaliação da saúde reflita uma percepção integrada do indivíduo, que inclua as dimensões biológicas, psicológicas e sociais ${ }^{21}$.

Em suma, os dados apresentados evidenciam que a população percebe saúde não apenas como ausência de doença, mas também como um constructo relacionado com aspectos sociais e demográficos, e em menor magnitude, com aspectos comportamentais. Portanto, abordagens em saúde devem reconhecer esse fato e superar o modelo simplista onde a saúde é dicotomizada em doente e não-doente.

\section{Colaboradores}

FF Reichert concebeu a ideia, realizou as análises e redigiu o manuscrito. MR Loch contribuiu na escrita e revisão bibliográfica. MF Capilheira participou da coleta dos dados e escrita do texto. Todos os autores aprovaram a versão final do artigo. 


\section{Referências}

1. Sundquist J, Johansson SE. Self reported poor health and low educational level predictors for mortality: a population based follow up study of 39.156 people in Sweeden. J Epidemiol Community Health 1997; 51(1):35-40.

2. Fonseca SA, Blank VLG, Barros MVG, Nahas MV Percepção de saúde e fatores associados em industriários de Santa Catarina, Brasil. Cad Saude Publica 2008; 24(3):567-576.

3. Szwarcwald CL, Souza-Junior PR, Esteves MA, Damacena GN, Viacava F. Socio-demographic determinants of self-rated health in Brazil. Cad Saude Publica 2005; 21(Supl. 1):54-64.

4. Franks P, Gold MR, Fiscella K. Sociodemographics, self-rated health, and mortality in the US. So Sci Med 2003; 56(12):2505-2514.

5. Lindstrom M, Sundquist J, Ostergren PO. Ethnic differences in self reported health in Malmo in southern Sweden. J Epidemiol Community Health 2001; 55(2):97-103.

6. Brasil. Ministério da Saúde (MS). VIGITEL Brasil 2006: Vigilância de fatores de risco e proteção para doenças crônicas por inquérito telefônico: estimativas sobre freqüência e distribuição sócio demográfica de fatores de risco e proteção para doenças crônicas nas capitais dos 26 estados brasileiros e no Distrito Federal em 2006. Brasília: MS; 2007.

7. Sousa TFS, Silva KS, Garcia LMT, Del Duca GF, Oliveira ESA, Nahas MV. Autoavalição de saúde e fatores associados em adolescentes do Estado de Santa Catarina, Brasil. Rev Paul Pediatr 2010; 28(4): 333-339.

8. Loch MR, Possamai CL. Associação entre percepção de saúde e comportamentos relacionados à saúde em adolescentes escolares de Florianópolis, SC. Cienc Cuid Saúde 2007; 6(Supl. 2):377-383.

9. Souza EM, Abrão FPS, Motta IA, Almeida JO. Autopercepção do estado de saúde: um estudo de prevalência com adolescentes de Ceilândia, Distrito $\mathrm{Fe}$ deral, Brasil. Comum Ciên Saúde 2006; 17(1):9-15.

10. Alves LS, Rodrigues RN. Determinantes da autopercepção de saúde entre idosos do Município de São Paulo, Brasil. Rev Panam Salud Publica 2005, 17(5/6):333-341.

11. Lima-Costa MF, Firmo JOA, Uchôa E. A estrutura da auto-avaliação da saúde entre idosos: Projeto Bambuí. Rev Saude Publica 2004; 38(6):827-834.

12. Associação Brasileira de Empresas de Pesquisa (ABEP). Critério de Classificação Econômica Brasil. São Paulo: ABEP; 2008
13. Craig CL, Marshall AL, Sjostrom M, Bauman AE, Booth ML, Ainsworth BE, Pratt M, Ekelund U, Yngve A, Sallis JF, Oja P. International physical activity questionnaire:12-country reability and validity. Med Sci Sports Exerc 2003; 35(8):1381-1395.

14. World Health Organization (WHO). Physical status: the use and interpretation of anthropometry. Report of a WHO Expert Committee. Geneva: World Health Organ Tech Rep Ser; 1995.

15. Instituto Brasileiro de Geografia e Estatística (IBGE) Censo 2000. [site na Internet]. [acessado 2012 out 15]. Disponível em: http://www.ibge. com.br/home/ estatistica/populacao/default_censo_2000.shtm

16. Barnes LL, Mendes de Leon CF, Bienias JL, Evans DA. A longitudinal study of black-white differences in social resources. J Gerontol B Psychol Sci Soc Sci 2004; 59(3):146-153.

17. Barros FC, Victora CG, Horta BL. Ethnicity and infant health in Southern Brazil. A birth cohort study. Int J Epidemiol 2001; 30(5):1001-1008.

18. Hernàndez $\mathrm{M}$, Herrero R, Sànchez $\mathrm{C}$, Montagut L. Autopercepción de salud en ancianos no institucionalizados. Atención Primaria 2001; 28(2):91-96.

19. Garbin CAS, Garbin AJI, Moimaz SAS, Gonçalves PE. A saúde na percepção do adolescente. Physis 2009; 19(1):227-238.

20. Fonseca MGUP, Firmo JOA, Loyola Filho AI, Uchô E. Papel da autonomia na auto-avaliação da saúde do idoso. Rev Saude Publica 2010; 44(1):159-165.

21. Jylhä M, Guralnik JM, Ferrucci L, Jokela J, Heikkinen E. Is self-rated health comparable across cultures and genders? J Gerontol B Psychol Sci Soc Sci 1998; 53(3):S144-S152.

Artigo apresentado em 22/06/2011

Aprovado em 28/07/2011

Versão final apresentada em 04/10/2011 